\title{
Comparison of 3T and 7T Susceptibility-Weighted Angiography of the Substantia Nigra in Diagnosing Parkinson Disease
}

\author{
M. Cosottini, D. Frosini, I. Pesaresi, G. Donatelli, P. Cecchi, M. Costagli, L. Biagi, R. Ceravolo, U. Bonuccelli, and M. Tosetti
}

\begin{abstract}
BACKGROUND AND PURPOSE: Standard neuroimaging fails in defining the anatomy of the substantia nigra and has a marginal role in the diagnosis of Parkinson disease. Recently 7T MR target imaging of the substantia nigra has been useful in diagnosing Parkinson disease. We performed a comparative study to evaluate whether susceptibility-weighted angiography can diagnose Parkinson disease with a 3T scanner.
\end{abstract}

MATERIALS AND METHODS: Fourteen patients with Parkinson disease and 13 healthy subjects underwent MR imaging examination at $3 T$ and 7T by using susceptibility-weighted angiography. Two expert blinded observers and 1 neuroradiology fellow evaluated the 3T and 7T images of the sample to identify substantia nigra abnormalities indicative of Parkinson disease. Diagnostic accuracy and intra- and interobserver agreement were calculated separately for 3T and 7T acquisitions.

RESULTS: Susceptibility-weighted angiography 7T MR imaging can diagnose Parkinson disease with a mean sensitivity of 93\%, specificity of $100 \%$, and diagnostic accuracy of $96 \%$. 3T MR imaging diagnosed Parkinson disease with a mean sensitivity of $79 \%$, specificity of $94 \%$, and diagnostic accuracy of $86 \%$. Intraobserver and interobserver agreement was excellent at 7T. At 3T, intraobserver agreement was excellent for experts, and interobserver agreement ranged between good and excellent. The less expert reader obtained a diagnostic accuracy of $89 \%$ at $3 \mathrm{~T}$.

CONCLUSIONS: Susceptibility-weighted angiography images obtained at 3T and 7T differentiate controls from patients with Parkinson disease with a higher diagnostic accuracy at 7T. The capability of 3T in diagnosing Parkinson disease might encourage its use in clinical practice. The use of the more accurate 7T should be supported by a dedicated cost-effectiveness study.

ABBREVIATIONS: HS = healthy subjects; PD = Parkinson disease; SN = substantia nigra; SWAN = susceptibility-weighted angiography; UHF = ultra-high-field

$\mathbf{P}$ arkinson disease (PD) is a common neurodegenerative disease whose pathologic substrate is nigrostriatal dopaminergic degeneration due to the neuronal loss in the pars compacta of the substantia nigra $(\mathrm{SN}){ }^{1}$

Received May 31, 2014; accepted after revision August 29.

From the IMAGO7 Foundation (M. Cosottini, M. Costagli), Pisa, Italy; Department of Translational Research and New Technologies in Medicine and Surgery (M. Cosottini, G.D.) and Neurology Unit (D.F., R.C., U.B.), Department of Clinical and Experimental Medicine, University of Pisa, Pisa, Italy; Neuroradiology Unit (I.P., P.C.), Department of Diagnostic and Interventional Radiology, Azienda Ospedaliero Universitaria Pisana, Pisa, Italy; and Stella Maris Scientific Institute (L.B., M.T.), Pisa, Italy.

The study is part of an experimental protocol named "Clinical Impact of UltraHigh-Field MRI in Neurodegenerative Diseases Diagnosis," RF-2009-1546281, approved and funded by Italian Ministry of Health and co-funded by the Health Service of Tuscany.

Please address correspondence to Mirco Cosottini, MD, Via Paradisa 2, 56124 Cisanello Pisa, Italy; e-mail: mircocosottini@libero.it

-- Indicates open access to non-subscribers at www.ajnr.org

7 Indicates article with supplemental on-line photo.

http://dx.doi.org/10.3174/ajnr.A4158
On the basis of the correlation between MR signal intensity at conventional field strengths and Perls staining for iron distribution, the medial portion of the midbrain with lower MR signal is attributed to the pars reticulata of the substantia nigra, and the lateral region (with higher MR signal), to the substantia nigra pars compacta. ${ }^{2}$ However, Perls staining and T2WI signal hypointensity do not match precisely, ${ }^{3}$ and the hypointense area on T2WI does not match the substantia nigra pars reticulata. ${ }^{4}$ Moreover, conventional MR imaging techniques, including segmented inversion recovery ratio imaging, ${ }^{5}$ fail to distinguish the inner structure of the substantia nigra. ${ }^{6}$ More advanced and recently proposed SN-derived biomarkers such as relaxometry, ${ }^{7,8} \mathrm{DTI},{ }^{9}$ and neuromelanin imaging ${ }^{10}$ are currently not yet accepted in evaluating patients with PD in clinical practice. ${ }^{11}$

Recently, by using high-resolution 3D susceptibility-weighted angiography (SWAN), ${ }^{12}$ the ultra-high-field (UHF) anatomy of the SN with its inner organization has been described ${ }^{13}$ as a 3-layer structure of different signal intensities along the posterior- 
anterior axis of the midbrain, resembling the dorsal and ventral components of the substantia nigra pars compacta and the substantia nigra pars reticulata, respectively. By using calbindin immunostaining, one can distinguish calbindin-positive (matrix) and calbindin-negative structures (nigrosomes) ${ }^{14}$ within the substantia nigra. In a recent MR imaging study at $7 \mathrm{~T},{ }^{15}$ nigrosome 1 , the largest and highly attenuated cluster of calbindin-negative neurons within the substantia nigra pars compacta ventralis, corresponded to the round hyperintense area observed in the intermediate and lateral portion of the substantia nigra pars compacta. ${ }^{16}$

In patients with $\mathrm{PD}$ the 3-layer organization and the hyperintense lateral spot within the SN (nigrosome 1) are lost, and this radiologic sign distinguishes patients with $\mathrm{PD}$ from healthy subjects (HS) on an individual basis with high accuracy. ${ }^{13}$

The diagnostic gain provided by $7 \mathrm{~T}$ imaging is a prerequisite for the clinical acceptance of UHF, but until now, 7T MR imaging examinations have been confined to the research environment. The neuroimaging-based diagnosis of PD might constitute an important addition to the clinical diagnosis of extrapyramidal disorders. Therefore, the diagnostic role of SWI performed with a clinical MR imaging scanner at 3T has been tested recently, ${ }^{17,18}$ with promising results. A direct comparison of $3 \mathrm{~T}$ and $7 \mathrm{~T}$ evaluation of the $\mathrm{SN}$ is mentioned in a pilot experience including 2 patients with PD. ${ }^{15}$

Here, we describe a comparative study with a case control design that prospectively evaluates the diagnostic accuracy of SWAN at 3T and 7T.

\section{MATERIALS AND METHODS \\ Subjects}

Fourteen patients with PD according to the clinical criteria for probable PD of the UK Parkinson's Disease Society Brain Bank ${ }^{19}$ were consecutively enrolled from a tertiary movement disorder center.

Patients ( 6 women and 8 men; mean age, 57.4 years; range, 39-69 years) had a disease mean duration at the moment of enrollment of 58 months (range, 6-120 months); the range of Hoehn and Yahr Scale ${ }^{20}$ was 1-2, and the motor part of the Unified Parkinson Disease Rating Scale ${ }^{21}$ was 18.3 (range, 9-37).

Thirteen right-handed HS (4 women and 9 men; mean age, 54.8 years; range, $41-66$ years) were enrolled to test the diagnostic accuracy of the SN MR imaging examination in discriminating patients with PD from controls. All HS were volunteers and had no personal or family history of psychiatric and neurologic disorders; their general and neurologic examination findings were unremarkable.

All patients and controls gave their informed consent to the enrollment and diagnostic procedures on the basis of the adherence to an experimental protocol called "Clinical Impact of UltraHigh-Field MRI in Neurodegenerative Diseases Diagnosis," RF2009-1546281, approved and funded by the Italian Ministry of Health and co-funded by the Health Service of Tuscany. The local competent ethics committee approved the study.

\section{MR Imaging Acquisition}

MR imaging examinations were performed in patients and controls by using a Discovery MR 950 7T imaging scanner (GE
Healthcare, Milwaukee, Wisconsin) equipped with a 2CH-TX/ 32CH-RX head coil (Nova Medical, Wilmington, Massachusetts) and a Discovery MR 7503 T scanner (GE Healthcare) equipped with an 8-channel head coil. Patients and controls underwent both $7 \mathrm{~T}$ and $3 \mathrm{~T}$ examination in a random order but within 1 month of recruitment.

The same technicians optimized the $3 \mathrm{D}$ multiecho gradientrecalled sequence (SWAN) at $7 \mathrm{~T}$ and $3 \mathrm{~T}$.

The MR imaging parameters at $7 \mathrm{~T}$ were $\mathrm{TE}=5.57,10.7,15.84$, $20.97,26.1,31.23,36.36,41.5 \mathrm{~ms}$; $\mathrm{TR}=55.7 \mathrm{~ms}$; flip angle $=8^{\circ}$; $\mathrm{NEX}=0.67$; in-plane resolution $=312 \mu \mathrm{m}$; thickness $=1.2 \mathrm{~mm}$; 18 partitions; receiver bandwidth $=67 \mathrm{KHz}$.

The MR imaging parameters at $3 \mathrm{~T}$ were $\mathrm{TE}=13,17.96,22.93$, $27.89,32.86,37.82,42.78,47.75,52.71,57.68,62.64 \mathrm{~ms}$; TR $=67.2$ $\mathrm{ms}$; flip angle $=15^{\circ} ; \mathrm{NEX}=0.68$; in-plane resolution $=394 \mu \mathrm{m}$; thickness $=1.2 \mathrm{~mm} ; 18$ partitions; receiver bandwidth $=62.5$ $\mathrm{KHz}$.

The SWAN acquisitions on both MR imaging systems targeted the midbrain and were oriented perpendicularly to the fourth ventricle floor.

The final output image was reconstructed into a $512 \times 512 \times$ 18 matrix by averaging the images obtained for each single echo. The SWAN acquisition time was 4 minutes 2 seconds at 7T and 4 minutes 20 seconds at $3 \mathrm{~T}$.

\section{Image Analysis}

According to the methodology of a previous study, ${ }^{13}$ the SN anatomy was evaluated along the rostrocaudal axis at the level of the inferior third of the red nucleus (level I) and at the level of the superior cerebellar peduncle decussation (level II), where the signal changes were more informative for diagnosing PD. Anatomy was considered normal if the $\mathrm{SN}$ at level I was characterized by a medial homogeneous hypointense region and by a lateral hyperintense spot between 2 hypointense layers and if a trilaminar organization characterized by a central hyperintense layer between 2 hypointense laminae was detectable at level II.

The criteria for abnormal SN anatomy were the loss of the hyperintense lateral spot at level I or/and the loss of the 3-layer organization at level II on at least 1 side of the midbrain.

Two experienced neuroradiologists were invited to identify "abnormal" SN in SWAN-targeted images acquired in the sample of patients and controls. Images acquired at $7 \mathrm{~T}$ and $3 \mathrm{~T}$ were separately loaded into a data base and presented in a blinded and randomized fashion. This process ensured that the readers were unaware of the clinical diagnosis. Readers were asked to identify patients with PD according to the "abnormal" appearance of the $\mathrm{SN}$. Images of each subject were presented twice after an interval of 7 days to calculate intra- and interrater reliability separately for $7 \mathrm{~T}$ and $3 \mathrm{~T}$ imaging. Intra- and interobserver reliability of responses from each reader were cross-tabulated to enable the calculation of agreement and the Cohen $\kappa$ statistic. ${ }^{22}$ The strength of agreement was considered low for $\kappa$ values of $0.21-$ 0.40 , moderate for $\kappa$ values of $0.41-0.60$, good for $\kappa$ values of $0.61-0.80$, and excellent for $\kappa$ values of $\geq 0.81$. Sensitivity, specificity, positive predictive value, negative predictive value, and diagnostic accuracy with respect to the clinical diagnosis were calculated. 
Sensitivity, specificity, PPV and NPV, diagnostic accuracy, and true-positive/true-negative results for each reader in each reading session

\begin{tabular}{|c|c|c|c|c|c|c|c|c|c|c|c|c|}
\hline & \multicolumn{6}{|c|}{$7 T$} & \multicolumn{6}{|c|}{$3 T$} \\
\hline & R1 1st & R1 2nd & R2 1st & R2 2nd & R3 lst & R3 2nd & R1 lst & $\mathrm{R} 1$ 2nd & R2 1st & R2 2nd & R3 lst & R3 2nd \\
\hline Sensitivity & 93 & 93 & 93 & 93 & 77 & 86 & 79 & 79 & 71 & 86 & 79 & 79 \\
\hline Specificity & 100 & 100 & 100 & 100 & 100 & 100 & 92 & 100 & 92 & 92 & 100 & 100 \\
\hline PPV & 100 & 100 & 100 & 100 & 100 & 100 & 92 & 100 & 91 & 92 & 100 & 100 \\
\hline NPV & 93 & 93 & 93 & 93 & 81 & 87 & 80 & 81 & 75 & 86 & 81 & 81 \\
\hline Accuracy & 96 & 96 & 96 & 96 & 85 & 93 & 85 & 89 & 81 & 89 & 89 & 89 \\
\hline $\mathrm{TP} / \mathrm{TN}$ & $13 / 13$ & $13 / 13$ & $13 / 13$ & $13 / 13$ & $10 / 13$ & $12 / 13$ & $11 / 12$ & $11 / 13$ & $10 / 12$ & $12 / 12$ & 11/13 & $11 / 13$ \\
\hline
\end{tabular}

Note:-R indicates reader; TP, true-positive; TN, true-negative; 1st, first reading; 2nd, second reading; NPV, negative predictive value; PPV, positive predictive value.

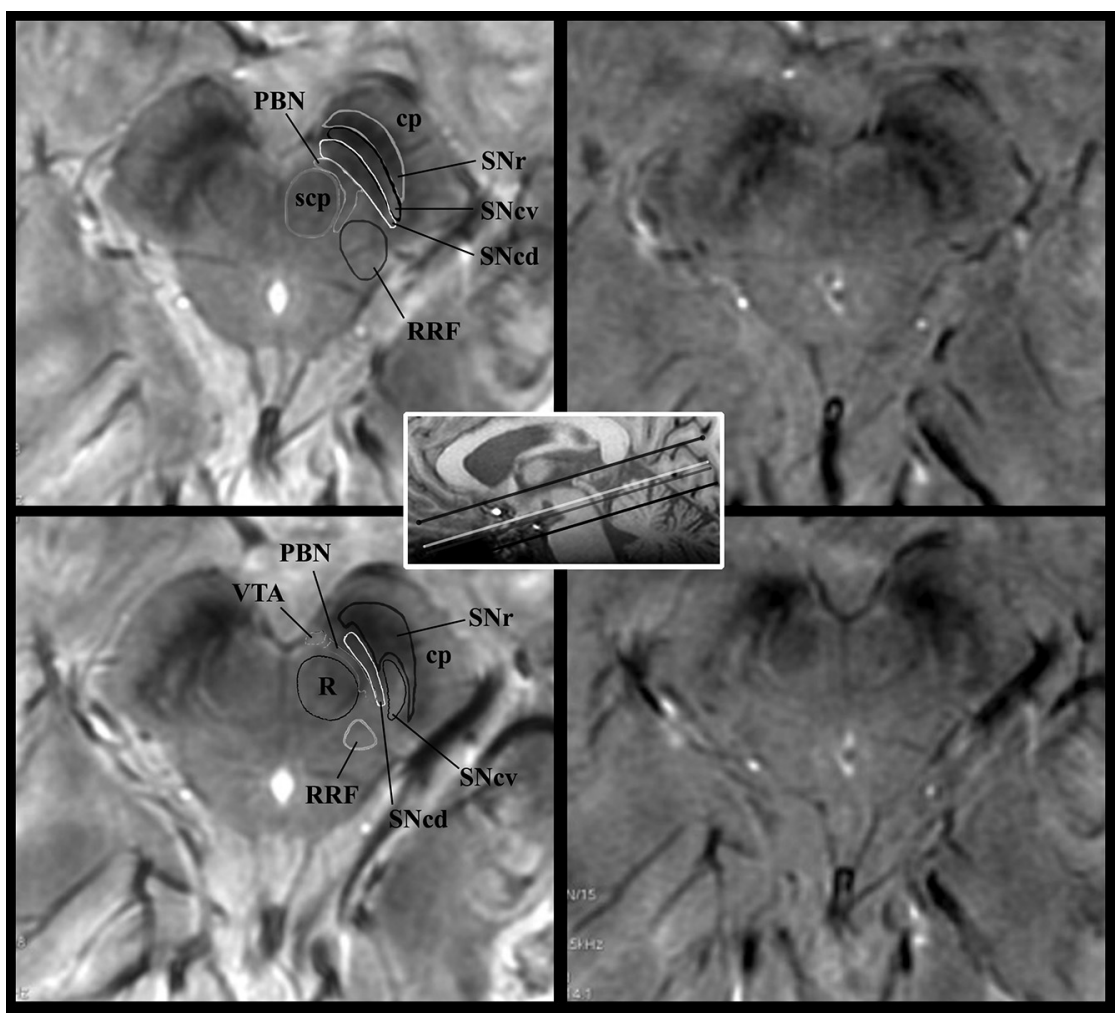

FIG 1. SWAN-targeted axial image of the midbrain in a healthy subject evaluated at $3 T$ (right column) and at 7T (left column). The trilaminar organization of the SN at level II (upper row) and the nigrosome formation at level I (lower row) are clearly shown with 3T and 7T magnets. Levels I and II of image acquisition are represented by white and gray lines in the scout image. On 7T images, we overlaid a diagram of the trilaminar structure of the SN derived by anatomic atlases. ${ }^{41}$ The diagnostic accuracy is elevated for both high- and ultra-high-fieldstrength magnets. cp indicates cerebral peduncle; PBN, parabrachial nucleus; RRF, retrorubral field; scp, superior cerebellar peduncle; SNcv, substantia nigra pars compacta ventralis; SNcd, substantia nigra pars compacta dorsalis; SNr, substantia nigra pars reticularis; VTA, ventral tegmental area; $\mathrm{R}$, red nucleus.

To test the role of radiologic signs in diagnosing $\mathrm{PD}$ and to reduce the bias of previous experiences at high and ultra-highfield strength magnets, we presented the entire data base of patients and controls to a third blinded observer with 5 years' experience in radiology at our university and whose experience in the neuroradiology unit included CT and 1.5T MR imaging studies of patients with neurodegenerative and movement disorders but not with acquisitions on 3T or 7T MR imaging systems. The third rater was invited to study the $\mathrm{SN}$ anatomy and its changes according to the most recent literature ${ }^{13,15}$ regarding the semiology of the SN in PD. A dataset consisting of 8 midbrain images at 3T (4 HS) and at 7T (4 HS) was used for training purposes. The images belonging to this training dataset were not included in the rest of this study. After training, the third rater evaluated all the $3 \mathrm{~T}$ and $7 \mathrm{~T}$ midbrain images previously presented to the 2 experienced neuroradiologists in a blinded and randomized fashion at 2 different times to calculate sensitivity, specificity, accuracy, and intraobserver agreement for $3 \mathrm{~T}$ and $7 \mathrm{~T}$ acquisitions.

\section{RESULTS}

The diagnostic accuracy, sensitivity, specificity, positive predictive value, and negative predictive value for each rater in each reviewing session are separately reported in the Table. In the next 2 paragraphs, the diagnostic parameters are reported as the mean value obtained by the 2 expert raters in the 2 reviewing sessions.

\section{T MR Imaging of the SN}

Sensitivity, specificity, positive predictive value, and negative predictive value for the diagnosis of PD were 93\%, 100\%, $100 \%$, and $93 \%$, respectively. Diagnostic accuracy was $96 \%$. Intraobserver agreement $(\kappa=1)$ and interobserver agreement $(\kappa=1)$ were excellent.

\section{T MR Imaging of the SN}

Sensitivity, specificity, positive predictive value, and negative predictive value for the diagnosis of PD were 79\%, 94\%, $94 \%$, and $81 \%$, respectively. Diagnostic accuracy was $86 \%$. At $3 \mathrm{~T}$, intraobserver agreement was excellent for both raters $(\kappa=0.924$ for rater 1 and $\kappa=0.851$ for rater 2 ) and interobserver agreement was good for the first reviewing session $(\kappa=0.773)$ and excellent for the second reviewing session $(\kappa=0.851)$.

\section{Performance of the Third, Less Expert Rater}

For the third rater, sensitivity, specificity, positive predictive value, negative predictive value, and accuracy were $82 \%, 100 \%$, $100 \%, 84 \%$, and $89 \%$ at $7 \mathrm{~T}$ and $79 \%, 100 \%, 100 \%, 81 \%$, and $89 \%$ at $3 \mathrm{~T}$, respectively. Intraobserver agreement was good $(\kappa=0.773)$ at $7 \mathrm{~T}$ and excellent $(\kappa=1)$ at $3 \mathrm{~T}$.

Examples of 3T correct, false-positive, and false-negative readings as classified by rater 2 are shown respectively in Figs 1-3. 


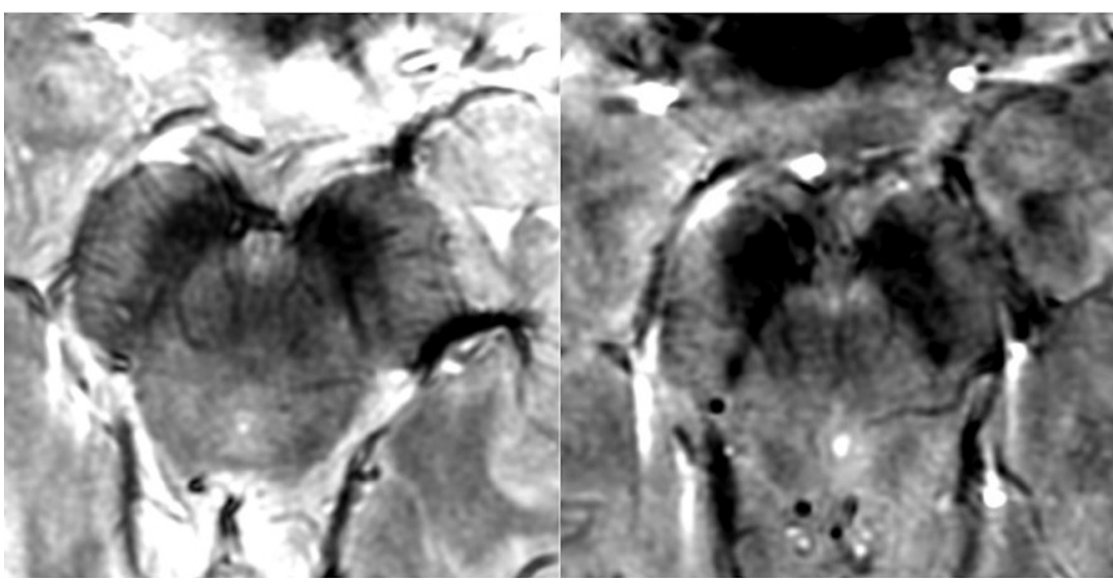

FIG 2. SWAN-targeted axial image of the midbrain in a healthy subject evaluated at $3 T$ (right) and 7T (left). The nigrosome formation appreciable at $7 \mathrm{~T}$ is interpreted as lost at 3T, generating a false-positive.

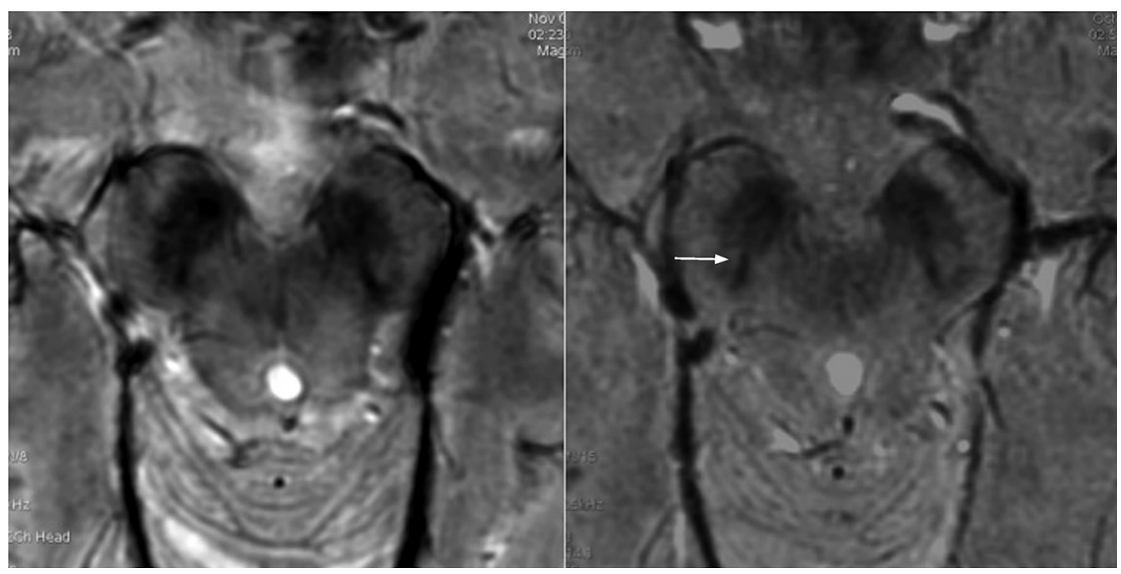

FIG 3. SWAN-targeted axial image of the midbrain in a patient with PD evaluated at $3 T$ (right) and 7T (left). The nigrosome formation seems recognizable in the left SN. On the right SN, the nigrosome formation is lost at 7T, while at 3T the slight hyperintense component (arrow) has been erroneously interpreted as a nigrosome, leading to a false-negative finding.

\section{DISCUSSION}

Our results confirm the capability of 7T MR imaging to reveal, through visual inspection of SWAN images, the loss of the 3-layer organization ${ }^{13}$ and of the lateral bright spot of the $\mathrm{SN}^{13,15,16}$ in patients with $\mathrm{PD}$, allowing high diagnostic accuracy.

MR imaging at $7 \mathrm{~T}$ is being tested in different clinical conditions $^{23}$ to assess its clinical importance for specific situations. ${ }^{24}$ UHF MR imaging examinations provide encouraging results in terms of tolerability and acceptance. ${ }^{25,26}$ At present, around the world, there are approximately 40 UHF MR imaging systems that are still confined to the research environment. In this study, we transferred the semiology adopted at $7 \mathrm{~T}$ in diagnosing $\mathrm{PD}$ to $3 \mathrm{~T}$ and verified that the diagnostic gain permitted by UHF could be extended, at least to some relevant extent, to a clinical scanner that is more easily accessible for patient care (Fig 1).

With high-resolution target imaging at 3T, we detected a partition of the SN in HS and identified patients with PD as reported in other recent experiences. ${ }^{17,18}$ Nevertheless as demonstrated by the lower intra- and interobserver agreement at 3T, the confidence in revealing the inner structure of the $\mathrm{SN}$ was inferior to that of 7T. The loss of the 3-layer organization of the SN or the loss of the hyperintense spot in the lateral component of the intermediate tier of the $\mathrm{SN}$ is less evident at $3 \mathrm{~T}$ and leaves some uncertainty in defining the pathologic aspect of the SN in PD. As demonstrated by the higher number of falsepositives and false-negatives at 3T, identifying the hyperintense central component of the normal SN (Fig 2) is more difficult, and the raters tended to overestimate subtle SN hyperintensities, which were misinterpreted as normal (Fig 3). The global effect is the reduction of the diagnostic accuracy of SWAN sequences at 3T with respect to 7T.

Because the MR imaging signal increases quadratically with the field strength while noise increases only linearly, the SNR is theoretically more than double at $7 \mathrm{~T}$ with respect to $3 \mathrm{~T}$, and this difference can be exploited to increase the spatial resolution. ${ }^{27}$ In our protocol, the 7T and 3T images have a similar spatial resolution $(312 \mu \mathrm{m}$ at $7 \mathrm{~T}$ versus 364 $\mu \mathrm{m}$ at $3 \mathrm{~T})$; therefore, this is unlikely to be the reason for the better performance of $7 \mathrm{~T}$. The superior imaging performance of 7T is more likely from the increased $\mathrm{T} 2{ }^{\star}$ contrast and magnetic susceptibility effects of paramagnetic substances, which are enhanced at higher magnetic fields. ${ }^{28-30}$ The magnetic susceptibility effects of iron contained in the SN scale linearly with magnetic field strength. At 7T, the larger susceptibility effect compared with $3 \mathrm{~T}$ results in more informative images of the midbrain with an increased contrast between the 2 hypointense iron-containing components of the $\mathrm{SN}$ and the intermediate hyperintense part that is preserved in HS (On-line Figure).

From a clinical point of view, a diagnostic error occurs in $10 \%-25 \%$ of patients with parkinsonism; and $20 \%$ of patients with PD coming to medical attention are not diagnosed as such. ${ }^{31}$ These diagnoses result in delayed or inappropriate therapy initiation. ${ }^{32}$ The accuracy of the clinical diagnosis of PD is $90 \%$ in a 5 -year observation period, ${ }^{33}$ and the rate of re-diagnosis from PD to an alternative disease is $5.9 \% .^{34}$ The most frequent wrong differential diagnosis is related to atypical parkinsonism ${ }^{35}$ but also to nondegenerative movement disorders such as essential tremor, dystonic tremor, and vascular pseudoparkinsonism. ${ }^{36}$ More rare but crucial misdiagnoses are normal pressure hydrocephalus ${ }^{37}$ and neoplastic lesions. ${ }^{38}$ The total annual cost of anti-Parkinson medication for these misdiagnosed patients has been estimated at $\mathfrak{E} 13,400$ (US $\$ 21,722$ ). ${ }^{34}$ The potential role of MR imaging would be to provide an earlier and more accurate diagnosis than the standard clinical assessment, improving the cost-effectiveness. The diagnostic accuracy of SWAN imaging at $3 \mathrm{~T}$ is lower than that 
at 7T but is still high enough to suggest a role in a clinical setting. Because the sensitivity of the clinical evaluation is high (96\%) and the specificity of the 3T MR imaging is higher than that of the clinical evaluation in a 5-year period $(82 \%),{ }^{36} 3 \mathrm{~T}$ MR imaging can be used in clinical practice as a confirmatory method. In a population with a clinical suspicion of $\mathrm{PD}$, the high specificity of 3T MR imaging allows identifying true-negative subjects, similar to nuclear medicine with radiotracers for dopamine transporters. ${ }^{39}$ Because MR imaging is a fundamental step in excluding pseudoparkinsonism or atypical parkinsonism, ${ }^{40}$ the use of a $3 \mathrm{~T}$ clinical scanner, including the imaging of the $\mathrm{SN}$, seems to be justified in extrapyramidal disorders.

Unfortunately SN imaging at 3T is less effective than at 7T in identifying true-positive cases, attenuating its impact. We hypothesize that patients with high clinical suspicion of $\mathrm{PD}$ and negative imaging findings of the SN at 3T might benefit from an evaluation at 7T. A dedicated study would be necessary to calculate whether the use of the more accurate diagnosis obtained with 7T MR imaging is cost-effective compared with to the use of 3T MR imaging only.

Regarding the results of the less expert rater unbiased by previous UHF MR imaging experiences, the high diagnostic accuracy and intrarater reproducibility at $7 \mathrm{~T}$ and $3 \mathrm{~T}$ confirm the robustness of the radiologic semiology consisting of simple and reproducible signs. ${ }^{17}$

The diagnostic accuracy is inferior at $3 \mathrm{~T}$ in both expert and less expert raters, indicating that the confidence in expressing a diagnostic judgment depends more on the magnetic field strength than on the rater's ability.

Some major limitations should be taken into account. First, the number of enrolled patients is limited mostly due to the limited number of patients available for evaluation at 7T. Patients and controls are not perfectly matched, though they do not differ significantly. The sequence parameters (TE and flip angle) slightly differ between the $2 \mathrm{MR}$ imaging scanners. TEs are automatically determined by the software and cannot be set by the operators, while the flip angle at $7 \mathrm{~T}$ is reduced to limit the signal drop related to the T2 relaxation time shortening at UHF. Finally, although images were randomly presented, the quality of images obtained at $7 \mathrm{~T}$ and $3 \mathrm{~T}$ is clearly different, and the 2 datasets can be clearly recognized by the readers, who therefore cannot be considered blinded with respect to the scanner but only with respect to the subject's condition.

\section{CONCLUSIONS}

The experience acquired in the research environment by using 7T systems allowed us to develop and refine targeted MR imaging acquisitions for $3 \mathrm{~T}$ scanners, improving the role of imaging in the diagnosis of PD at a single-subject level in clinical practice. However if the higher diagnostic accuracy of SWAN at 7T with respect to $3 \mathrm{~T}$ could justify the use of the $7 \mathrm{~T}$ in clinical practice, a cost-effectiveness study to support this possibility should be performed.

\section{ACKNOWLEDGMENTS}

We wish to acknowledge the help provided by Mark Symms.
Disclosures: Ubaldo Bonuccelli-UNRELATED: Grants/Grants Pending: Regione Toscana Health Authority, for a Parkinson disease study (€200,000 [US \$253,165])*; Payment for Lectures (including service on Speakers Bureaus): Lundbeck, Chiesi, GlaxoSmithKline, Novartis, Comments: For speeches at meetings (€2000 [US \$2530]); Travel/Accommodations/Meeting Expenses Unrelated to Activities Listed: Lundbeck, Chiesi, ${ }^{*}$ GlaxoSmithKline, ${ }^{*}$ Novartis, ${ }^{*}$ Comments: for participation at neurology meetings (€2000 [US \$2530]). *Money paid to the institution.

\section{REFERENCES}

1. Fearnley JM, Lees AJ. Ageing and Parkinson's disease: substantia nigra regional selectivity. Brain 1991;114:2283-301

2. Drayer BP. Imaging of the aging brain. Part II. Pathologic conditions. Radiology 1988;166:797-806

3. Rutledge JN, Hilal SK, Silver AJ, et al. Study of movement disorders and brain iron by MR. AJR Am J Roentgenol 1987;149:365-79

4. Oikawa H, Sasaki M, Tamakawa $Y$, et al. The substantia nigra in Parkinson disease: proton density-weighted spin-echo and fast short inversion time inversion-recovery MR findings. AJNR Am J Neuroradiol 2002;23:1747-56

5. Hutchinson M, Raff U, Lebedev S. MRI correlates of pathology in parkinsonism: segmented inversion recovery ratio imaging (SIRRIM). Neuroimage 2003;20:1899-902

6. Minati L, Grisoli M, Carella F, et al. Imaging degeneration of the substantia nigra in Parkinson disease with inversion-recovery MR imaging. AJNR Am J Neuroradiol 2007;28:309-13

7. Ordidge RJ, Gorell JM, Deniau JC, et al. Assessment of relative brain iron concentrations using T2-weighted and T2*-weighted MRI at 3 Tesla. Magn Reson Med 1994;32:335-41

8. Martin WR, Wieler M, Gee M. Midbrain iron content in early Parkinson disease: a potential biomarker of disease status. Neurology 2008;70:1411-17

9. Vaillancourt DE, Spraker MB, Prodoehl, et al. High-resolution diffusion tensor imaging in the substantia nigra of de novo Parkinson disease. Neurology 2009;72:1378-84

10. Sasaki M, Shibata E, Tohyama K, et al. Neuromelanin magnetic resonance imaging of locus ceruleus and substantia nigra in Parkinson's disease. Neuroreport 2006;17:1215-18

11. Lehéricy S, Sharman MA, Dos Santos CL, et al. Magnetic resonance imaging of the substantia nigra in Parkinson's disease. Mov Disord 2012;27:822-30

12. Haacke EM, Xu Y, Cheng YC, et al. Susceptibility weighted imaging (SWI). Magn Reson Med 2004;52:612-18

13. Cosottini M, Frosini D, Pesaresi I, et al. MR imaging of the substantia nigra at $7 \mathrm{~T}$ enables diagnosis of Parkinson disease. Radiology 2014;271:831-38

14. Damier P, Hirsch EC, Agid Y, et al. The substantia nigra of the human brain. I. Nigrosomes and the nigral matrix, a compartmental organization based on calbindin $\mathrm{D}(28 \mathrm{~K})$ immunohistochemistry. Brain 1999;122:1421-36

15. Blazejewska AI, Schwarz ST, Pitiot A, et al. Visualization of nigrosome 1 and its loss in PD: pathoanatomical correlation and in vivo 7 T MRI. Neurology 2013;81:534-40

16. Kwon DH, Kim JM, Oh SH, et al. Seven-Tesla magnetic resonance images of the substantia nigra in Parkinson disease. Ann Neurol 2012;71:267-77

17. Schwarz ST, Afzal M, Morgan PS, et al. The "swallow tail" appearance of the healthy nigrosome: a new accurate test of Parkinson's disease-a case-control and retrospective cross-sectional MRI study at 3T. PLoS One 2014;9:e93814

18. Mueller C1, Pinter B, Reiter E, et al. Visualization of nigrosome 1 and its loss in PD: pathoanatomical correlation and in vivo 7T MRI. Neurology 2014;82:1752

19. Gibb WR, Lees AJ. The relevance of the Lewy body to the pathogenesis of idiopathic Parkinson's disease. J Neurol Neurosurg Psychiatry 1988;51:745-52

20. Hoehn MM, Yahr MD. Parkinsonism: onset, progression and mortality. Neurology 1967;17:427-42

21. Fahn S, Elton RL, members of the UPDRS Development Committee. 
Unified Parkinson's Disease Rating Scale. In: Fahn D, Marsden CD, Calne D, et al, eds. Recent Development in Parkinson's Disease. Vol. 2. Florham Park: Macmillan Healthcare Information; 1987

22. Landis JR, Koch GG. The measurement of observer agreement for categorical data. Biometrics 1977;33:159-74

23. van der Kolk AG, Hendrikse J, Zwanenburg JJ, et al. Clinical applications of 7 T MRI in the brain. Eur J Radiol 2013;82:708-18

24. Knopp MV. Clinical applications of ultra-high field 7T MR: moving to FDA/EU approval - potential clinical applications for ultra-high field MR. In: Proceedings of the International Society Magnetic Resonance Medicine Weekday Educational Course, Montreal, Quebec, Canada. May 11, 2011

25. Theysohn JM, Maderwald S, Kraff O, et al. Subjective acceptance of 7 Tesla MRI for human imaging. MAGMA 2008;21:63-72

26. Cosottini M, Frosini D, Biagi L, et al. Short-term side-effects of brain MR examination at 7 T: a single-centre experience. Eur Radiol 2014;24:1923-28

27. Kuhl CK, Traber F, Schild HH. Whole-body high-field-strength (3.0-T) MR imaging in clinical practice. Part I. Technical considerations and clinical applications. Radiology 2008;246:675-96

28. Haacke EM, Mittal S, Wu Z, et al. Susceptibility-weighted imaging: technical aspects and clinical applications, part 1. AJNR Am J Neuroradiol 2009;30:19-30

29. Haller S, Badoud S, Nguyen D, et al. Differentiation between Parkinson disease and other forms of Parkinsonism using support vector machine analysis of susceptibility-weighted imaging (SWI): initial results. Eur Radiol 2013;23:12-19

30. Wang Y, Butros SR, Shuai X, et al. Different iron-deposition patterns of multiple system atrophy with predominant parkinsonism and idiopathetic Parkinson diseases demonstrated by phase-corrected susceptibility-weighted imaging. AJNR Am J Neuroradiol 2012;33:266-73
31. Schrag A, Ben-Shlomo Y, Quinn N. How valid is the clinical diagnosis of Parkinson's disease in the community? J Neurol Neurosurg Psychiatry 2002;73:529-34

32. Jankovic J, Rajput AH, McDermott MP, et al. The evolution of diagnosis in early Parkinson disease. Arch Neurol 2000;57:369-72

33. Hughes AJ, Daniel SE, Lees AJ. Improved accuracy of clinical diagnosis of Lewy body Parkinson's disease. Neurology 2001;57:1497-99

34. Newman EJ, Breen K, Patterson J, et al. Accuracy of Parkinson's disease diagnosis in $\mathbf{6 1 0}$ general practice patients in the West of Scotland. Mov Disord 2009;24:2379-85

35. Hughes AJ, Daniel SE, Ben-Shlomo Y, et al. The accuracy of diagnosis of parkinsonian syndromes in a specialist movement disorder service. Brain 2002;125:861-70

36. Meara J, Bhowmick BK, Hobson P. Accuracy of diagnosis in patients with presumed Parkinson's disease. Age Ageing 1999;28:99-102

37. Curran T1, Lang AE. Parkinsonian syndromes associated with hydrocephalus: case reports, a review of the literature, and pathophysiological hypotheses. Mov Disord 1994;9:508-20

38. Frosini D, Ceravolo R, Rossi C, et al. Bilateral thalamic glioma presenting with parkinsonism. Mov Disord 2009;24:2168-69

39. Bajaj N, Hauser RA, Grachev ID. Clinical utility of dopamine transporter single photon emission CT (DaT-SPECT) with (123I) ioflupane in diagnosis of parkinsonian syndromes. J Neurol Neurosurg Psychiatry 2013;84:1288-95

40. Berardelli A, Wenning GK, Antonini A, et al. EFNS/MDS-ES/ENS recommendations for the diagnosis of Parkinson's disease. Eur J Neurol 2013;20:16-34

41. Halliday G, Reyes S, Double K. Substantia nigra, ventral tegmental area, and retrorubral fields. In: Main JK, Paxinos G, eds. The Human Nervous System. 3rd ed. London: Academic Press; 2012: $439-55$, chap 3 